\title{
FITOSOCIOLOGÍA URBANA I: LA COMUNIDAD DE POA ANNUA L. Y CORONOPUS DIDYMUS (L.) SM. DEL CENTRO DE ARGENTINA
}

\author{
Marta M. MOGLIA
}

\begin{abstract}
RESUMEN. Fitosociología urbana I: La comunidad de Poa annua L. y Coronopus didymus (L.) Sm. del centro de Argentina. Se comentan algunos de los problemas asociados a la práctica fitosociológica en zonas urbanizadas y, en este contexto, se analiza la identidad de una comunidad de Poa annua L. y Coronopus didymus (L.) Sm. presente en la zona central de Argentina. Los inventarios realizados en las provincias de Mendoza, San Luis, Córdoba y Buenos Aires, fueron comparados con los de las asociaciones relacionadas. Siguiendo el criterio para definir alianzas y asociaciones y utilizando Twinspan como método de análisis objetivo, se consideró a la comunidad como Bromo catharticiCoronopodetum didymi ass. nova, vicariante neotropical, chaqueña y estrechamente relacionada con el Poo annuae- Coronopodetum didymi Carretero \& Aguilella 1995, ibérico. Se propone la ampliación del areal, como neófita, de la alianza Policarpion tetraphylli Rivas-Martínez 1975 hasta América del Sur y se resalta la necesidad de incluir en sintaxones de amplia distribución a los taxones frecuentes en hábitats antropozoogénicos, lo cual facilitaría el desarrollo de la Fitosociología urbana a lo largo del mundo.
\end{abstract}

Palabras clave. Asociación, Policarpion tetraphylli, habitats antropozoogénicos, ciudades, Argentina

\begin{abstract}
Urban Phytosociology: The Poa annua L. and Coronopus didymus (L.) Sm. community of the center of Argentina. Some of the problems associated to the phytosociological practice in urbanized areas are commented. The identity of a Poa annua L. and Coronopus didymus (L.) Sm. community, of the central area of Argentina, is analyzed in this context. The relevés were taken in Mendoza, San Luis, Córdoba, and Buenos Aires provinces and they were compared with those of the related associations. On the basis of definitions for alliances and associations and using Twinspan, to achieve an objective analysis, the community recorded was considered as Bromo catharthici Coronopodetum didymi ass. nova. This is a vicariant (neotropical, chaquenian) and closely related with the iberic Poo annuae - Coronopodetum didymi Carretero \& Aguilella 1995 association. The amplification of the areal, as neophyte, of the alliance Policarpion tetraphylli Rivas-Martínez 1975 to South America is proposed, and the necessity to include into sintaxa of wide distribution to the frequent taxa of anthropozoogenic habitats is suggested, in order to facilitate the phytosociological development along the world.
\end{abstract}

Key words: Association, Policarpion tetraphylli, anthropozoogenic habitats, cities, Argentina 


\section{INTRODUCCIÓN Y OBJETIVOS}

La Ecología de las ciudades, o Ecología urbana, ha sido ampliamente estudiada en las últimas décadas (Florgård, 2004). Sin embargo, por algunos motivos que se comentan a continuación, la Fitosociología no ha tenido un papel relevante en esta nueva disciplina.

Varios autores señalan que el reconocimiento de comunidades vegetales en las ciudades, en el verdadero sentido fitosociológico, es decir, con indicación de las especies características, es problemático (Witting, 1989; Pysek, 1995; Trepl, 1995; Kent et al., 1999). También Rivas-Martínez (1977) expresó acerca de las comunidades ruderales y arvenses que «...Uno de los problemas más enrevesados en la Europa occidental es el de la vegetación ruderal y arvense... la falta de unanimidad de criterios, el modo diverso en la toma de inventarios y el diferente concepto en la definición de algunos sintaxones de rango superior, hace difícil cualquier ensayo tipológico...». Estos problemas no son sólo patrimonio europeo, sino que posiblemente sean comunes a la mayor parte de las regiones de la tierra donde se realiza Fitosociología. Los mismos se suscitan principalmente, debido a que en estas comunidades participan taxones de amplia distribución; como así también a consecuencia del enrevesado y complejo dinamismo que las caracteriza, profundamente influido por los factores antrópicos.

En Sudamérica esta disciplina ha tenido un desarrollo menos importante que en los países europeos. Una de las principales dificultades que debe afrontar quien trabaja en el tema, es el de la asignación de las comunidades o asociaciones detectadas a categorías de rango superior. Esto ocurre, en algunos casos, debido a que éstas no han sido aún definidas. En otros, como el que atañe a las comunidades nitrófilas, porque los taxones que deberían ser característicos de las mismas han sido asignados a sintaxones cuya corología no se corresponde con la del área de estudio y/o a que los sintaxones a los que deberían pertenecer las comunidades estudiadas por su cortejo específico, fenología, ecología, etc., han sido ligados a un areal restringido. Así, lo que normalmente sucede es que las asociaciones se dan a conocer como entidades aisladas, o como "comunidades de" y no relacionadas a un sistema jerárquico. En el peor de los casos se resuelve la creación de nuevos sintaxones, cuyos taxones característicos ya habían sido previamente asignados, dando como resultado un "caos fitosociológico" al que pocos investigadores se atreven a enfrentar.

El resultado de todo esto es que en la actualidad, debido a estos problemas y a la fuerte irrupción en las últimas décadas de la Ecología vegetal sustentada por la estadística convencional "más publicable», cada vez son menos los investigadores jóvenes que se atreven a la Fitosociología en estas regiones. Los problemas señalados son particularmente importantes en áreas urbanas, debido a que en ellas se concentra un conjunto de especies comunes a la mayor parte del globo, como resultado de una serie de condiciones ambientales que se repiten y de la creciente ampliación de las vías de comunicación entre áreas.

Ya, a mediados del siglo XX, Coquillat (1951) señaló que las especies más frecuentes en todo el globo son características de suelos sometidos a perturbaciones, principalmente en los situados próximos a aglomeraciones humanas. Una de las clases que incluye gran cantidad de taxones urbanícolas cosmopolitas y que se encuentra ampliamente representada en las ciudades de todo el mundo es Polygono-Poetea annuae Rivas Martínez 1975. Este sintaxon 
comprende a las comunidades de plantas anuales efímeras $y$, excepcionalmente perennes reptantes de tamaño reducido, de caminos de áreas urbanas y rurales, que se desarrollan en todo el mundo bajo climas desde templados a fríos, con lluvias estacionales (Rivas-Martínez et al. 2002). Entre sus especies características se incluyen algunas de las consideradas las más comunes de la tierra (Coquillat, op. cit.) como Poa annua y Polygonum aviculare. En las áreas urbanas de la zona central de Argentina, donde los climas son templados y las precipitaciones se concentran principalmente en verano, las comunidades de esta clase son frecuentes. No obstante, la clase no se cita en el trabajo preliminar de sintaxonomía de la vegetación ruderal de Argentina (Carretero, 1993).

En el marco de un estudio sobre las comunidades de la clase en la región, se realizaron inventarios de vegetación en un gradiente longitudinal, desde la provincia de Mendoza hasta la ciudad de Mar del Plata (Buenos Aires). En el transcurso del mismo se detectó una comunidad con caracteres similares al Poo anuae-Coronopodetum didymi Carretero y Aguilella 1995. Las preguntas que se suscitaron fueron ise trata de la misma asociación o es una vicariante de la misma? ¿Debería incluirse en el Polycarpion tetraphylly? La vegetación urbanícola ¿sigue patrones comunes en diferentes partes globo? La respuesta a estos interrogantes fueron los objetivos que se pretendieron alcanzar en el presente trabajo.

\section{MATERIAL Y MÉTODOS}

Área de estudio. El área de estudio (fig. 1, tab. 1) incluyó a las ciudades de Mendoza (prov. Mendoza), San Luis (prov. San Luis), Villa Mercedes (prov. San Luis), Río Cuarto (prov. Córdoba), La Plata (prov. Buenos Aires) y Mar del Plata (prov. Buenos Aires) de la República Argentina.

La distancia en línea recta desde Mendoza a Mar del Plata comprende unos $1150 \mathrm{~km}$ y corresponde aproximadamente al ancho máximo del país. Los climas son templados en toda el área, desde áridos en Mendoza, en el piedemonte de la precordillera andina, hasta húmedos en $\mathrm{La}$ Plata, próxima al Río de La Plata, y en Mar del Plata sobre la costa Atlántica.

Metodología. Durante los años 2002 a 2005 se realizaron inventarios de la comunidad en alcorques de aceras, bordes

\begin{tabular}{lllllll}
\hline Ciudad & Provincia & Latitud & Longitud & A & P & $\mathbf{T}^{\mathbf{o}}$ \\
\hline Mendoza & Mendoza & $32^{\circ} 55^{\prime}, \mathrm{S}$ & $68^{\circ} 51^{\prime}, \mathrm{O}$ & 760 & 235 & 15.5 \\
San Luis & San Luis & $33^{\circ} 16^{\prime} \mathrm{S}$ & $66^{\circ} 21^{\prime} \mathrm{O}$ & 716 & 588 & 17 \\
Villa Mercedes & San Luis & $33^{\circ} 41^{\prime} \mathrm{S}$ & $65^{\circ} 28^{\prime}, \mathrm{O}$ & 512 & 606 & 15.5 \\
Río Cuarto & Córdoba & $33^{\circ} 08^{\prime} \mathrm{S}$ & $64^{\circ} 08^{\prime} \mathrm{O}$ & 61 & 700 & 17.3 \\
La Plata & Buenos Aires & $34^{\circ} 55^{\prime}, \mathrm{S}$ & $5^{\circ} 57^{\prime}, \mathrm{O}$ & 15 & 950 & 16.3 \\
Mar del Plata & Buenos Aires & $37^{\circ} 56^{\prime} \mathrm{S}$ & $5^{\circ} 35^{\prime} \mathrm{O}$ & 21 & 1200 & 14 \\
\hline
\end{tabular}

Tabla 1. Ubicación, altitud y parámetros climáticos para cada ciudad. A: altitud (m s.n.m.), P: precipitación media anual $(\mathrm{mm}), \mathrm{T}^{\mathrm{o}}$ : temperatura media anual $\left({ }^{\circ} \mathrm{C}\right)$. A: altitude (m s..n.m.), P: Mean annual precipitation $(\mathrm{mm}), \mathrm{T}^{\circ}$ : Mean annual temperature $\left({ }^{\circ} \mathrm{C}\right)$ 

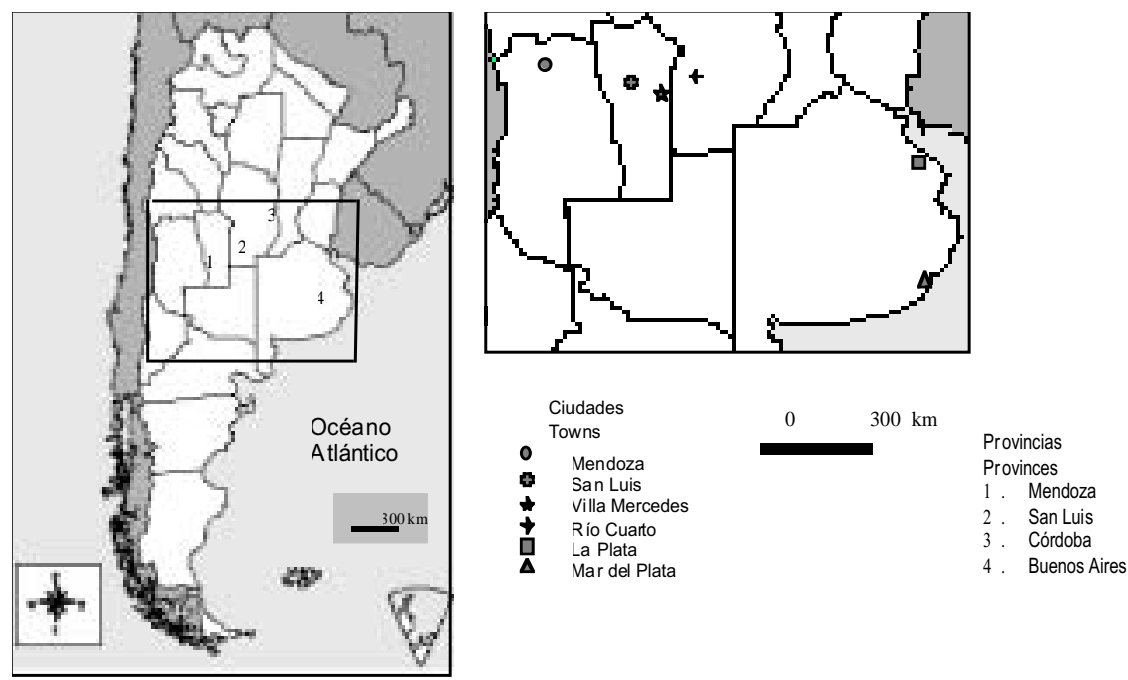

Figura 1. Mapas del área de estudio. Izquierda: provincias; derecha: ciudades. Maps of the study area. Left: Provinces; right: towns.

de caminos y zonas ajardinadas, siguiendo la metodología fitosociológica de la Escuela Zürich-Montpellier (Mueller-Dombois \& Ellemberg, 1974). Los mismos fueron posteriormente comparados con los originales del Poo anuae-Coronopodetum didymi Carretero \& Aguilella 1995 de la ciudad de Valencia.

El análisis se realizó teniendo en cuenta los caracteres señalados para definir alianzas y asociaciones (Géhu \& Rivas-Martínez, 1981; Rivas-Martínez, 1996; Weber et al., 2000). Los tres caracteres que todas las definiciones de asociación incluyeron explícitamente fueron: composición florística, fisonomía y características del hábitat, a los que se sumaron los estadísticos, biogeográficos, sucesionales, históricos y antropogénicos, en una o más de ellas.

Para la nomenclatura de los táxones citados en el trabajo se siguió a Zuloaga et al. (1994) y Zuloaga y Morrone (1996; 1999) y para la de los sintáxones a Rivas-Martínez et al. (2002).

Con el fin de lograr un análisis objetivo de los datos se conformó un análisis Twinspan: Análisis indicador de especies de dos vías (Hill, 1994) en el que se incluyeron, además de los inventarios de la comunidad hallada (BCD), los cinco del Poo annuaeCoronopodetum didymi (PCD), los tres del Poo annuae-Coronopodetum squamati (Oberdorfer 1957) Gutte 1966 (PCS) realizados en Valencia por Carretero y Aguilella (1995) y cuatro inventarios de una comunidad de Cotula australis (CC) registrada en zonas más húmedas y sombrías del área de estudio. Los inventarios de PCS, PCD y CC fueron resumidos con sus valores de presencia en una columna de la tabla 2 para cada comunidad. Si bien el número de inventarios para asignar valores de presencia fue menor de los seis requeridos (Géhu \& Rivas-Martínez, 1981), se presentaron de esta forma para mostrar en forma sintética la composición aproximada de los mismos. 


\section{RESULTADOS}

\section{Caracteres de la comunidad}

\section{Bromo cathartici-Coronopodetum didymi Moglia ass. nova}

[Tabla 2, holotypus ass; inv. 8]

\section{Florísticos, fisonómicos y} estadísticos. Se trata de una comunidad herbácea xerofítica, inverno-primaveral, con un estrato bajo dominante y baja riqueza específica. Se encuentra presidida por Coronopus didymus, que aporta la mayor proporción de biomasa y secundada por $P o a$ annua. Otras compañeras de importancia fueron Bromus catharticus y Cynodon dactylon. La primera de estas especies, nativa en Argentina, es muy frecuente en zonas urbanizadas del país, como así también en áreas similares de otras regiones templadas del mundo. La segunda es común en todas las comunidades de pisoteo de las ciudades del área, teniendo carácter invasor en gran parte del territorio argentino (Ruiz Leal, 1972) y en otras regiones del mundo (Marzocca, 1979). Entre sus acompañantes son también frecuentes terófitos de Stellarietea mediae como Sisymbrium irio, Sonchus oleraceus y Taraxacum officinale.

La superficie ocupada por la comunidad fue de dimensiones reducidas, si bien, en algunos sectores de la ciudad de San Luis, se presentó reiteradamente en cada uno de sus alcorques contiguos (tab. 2).

II. Sucesionales o dinámicos. Presenta carácter de colonizadora. En general no abandona el estadio inicial de la sucesión, debido a que los factores antropozoogénicos que la condicionan se mantienen relativamente constantes a través del tiempo, constituyendo una comunidad permanente. No obstante, en algunas ocasiones se observó un reemplazo estacional de la comunidad durante el verano (sinfenosucesión), por otra de la misma clase dominada por Alternanthera pungens.

III. Históricos y antropogénicos y del hábitat. Surge como producto del intenso efecto antropozoogénico en las áreas urbanizadas. Los hábitats en los que se desarrolla son principalmente los alcorques y bordes de caminos y aceras que generalmente presentan un alto grado de nitrificación, debido a que estos sitios son frecuentados por los perros, que con sus deyecciones elevan el contenido de nitratos del suelo. Además, el pisoteo producido por éstos y por los transeúntes origina una compactación superficial. No obstante los suelos son relativamente permeables al agua que reciben, principalmente a través del riego y de la limpieza de calles y aceras.

IV. Corológicos. La comunidad estuvo presente en toda el área investigada. Fue más frecuente hacia el oeste y centro, haciéndose más rara hacia el Este, donde los suelos son de texturas más finas, volviendo a reaparecer en la costa atlántica, sobre suelos con alto contenido de arena. Corológicamente se sitúa hasta el momento en la Región Neotropical, Dominio Chaqueño, en las Provincias del Monte (Mendoza capital), Chaco (San Luis capital), Espinal (Villa Mercedes y Río Cuarto) y Pampeana (La Plata y Mar del Plata) según el esquema fitogeográfico de Cabrera (1976).

\section{DISCUSIÓN}

Afinidades y diferencias con el Poo annuae-Coronopodetum didymi Carretero \& Aguilella 1995

I. Florísticos, fisonómicos y estadísticos. De la misma manera que la 
Tabla 2

1-11: Bromo cathartici-Coronopodetum didymi ass. nova., PCS: Poo annuaeCoronopodetum squamati, PCD: Poo annuae-Coronopodetum didymi, CC: Comunidad de Cotula australis (Polycarpion tetraphylli, Polygono arenastriPoetalia annuae, Polygono-Poetea annuae)

\begin{tabular}{|c|c|c|c|c|c|c|c|c|c|c|c|c|c|}
\hline $\mathrm{N}^{\circ}$ de orden & 1 & 2 & 3 & 4 & 5 & 6 & 7 & 8 & 9 & 10 & 11 & PCD & PCS \\
\hline Área $\left(m^{2}\right)$ & 1 & 1.5 & 1 & 2 & 1.5 & 2 & 1 & 4 & 4 & 2 & 1 & 3 & 5.7 \\
\hline Cobertura (\%) & 80 & 95 & 60 & 60 & 60 & 50 & 80 & 70 & 90 & 80 & 40 & 54 & 41.7 \\
\hline \multicolumn{14}{|l|}{$\begin{array}{l}\text { Características de } \\
\text { a sociación y unidades } \\
\text { superiores }\end{array}$} \\
\hline Coronopus didymus & 5 & 5 & 3 & 3 & 2 & 3 & 2 & 2 & 4 & 2 & 1 & V & IV \\
\hline Pоа аппиа & 1 & 2 & 2 & 2 & 2 & 1 & 4 & 1 & 2 & 4 & 2 & IV & IV \\
\hline Bromus catharticus & 1 & + & 1 & 1 & 2 & . & 1 & . & 1 & + . & 2 & . & . \\
\hline Cotula australis & . & . & . & . & . & . & + & 3 & 2 & + . & 1 & . & II \\
\hline Polygonum aviculare & 1 & . & . & . & . & . & . & . & . & . & . & . & . \\
\hline Alternanthera pungens & . & . & + & . & . & . & . & . & . & . & . & . & . \\
\hline Coronopus squamatus & . & . & . & . & . & . & . & . & . & . & . & . & $\mathrm{V}$ \\
\hline Polygonum arenastrum & . & . & . & . & . & . & . & . & . & . & . & . & II \\
\hline
\end{tabular}

Compañeras

Cynodon dactylon

Sisymbrium irio

Sonchus oleraceus

Taraxacum officinale

Chenopodium hircinum

Malva parviflora

Dichondra microcalyx

Lepidium bonariense

Amaranthus muricatus

Conyza bonariensis

Aster squamatus

Veronica persica

Euphorbia peplus

Oxalis corniculata

Cyperus rotundus

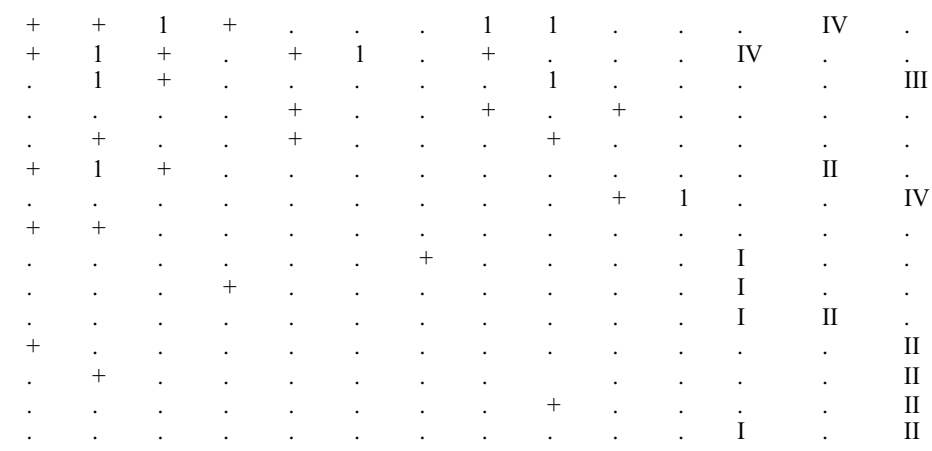

Otros táxones con + en un solo inventario: Inv. 1: Chenopodium multifidum, Bromus brevis. Inv. 7: Capsella bursa-pastoris y Amaranthus muricatus. Inv. 9: Oxalis corniculata. Otros táxones con I en Poo annuae-Coronopodetum didymi (PC): Convolvulus arvensis, Diplotaxis erucoides, Hordeum leporinum, Melilotus indicus, Picris echioides. Otros táxones con II en Poo annuae-Coronopodetun squamati (PCS): Lolium perenne. Otros táxones con II en la comunidad de Cotula australis community (CC): Geranium molle, Bowlesia incana, Parietaria debilis, Marrubium vulgare, Trifolium repens, Cyclospermum leptophyllum.

Localidades (provincia), hábitat, fecha. 1: Barrio El Lince, San Luis capital (San Luis), alcorque de acera, 26-09-02. 2: Barrio F. Bogado, San Luis Capital (San Luis), alcorque de acera, 26-09-02. 3: San Martín sur, San Luis capital (San Luis), margen de calle, 2-10-92. 4: Mar del Plata, ciudad (Buenos Aires), alcorque, 5-11-05. 5: Barrio Amep, San Luis capital (San Luis), borde de camino, 5-10-02. 6: Villa Mercedes, ciudad (San Luis), 15-08-03, margen de camino. 7: Rectorado, San Luis capital (San Luis), borde de camino, 12-09-02. 8: Barrio El Lince, San Luis capital (San Luis) parche en un césped de borde de acera, 26-09-02. 9: Río Cuarto, ciudad, (Córdoba), 10-11-03, margen de acera. 10: Mendoza, ciudad (Mendoza), alcorque de acera, 3-11-04. 11: City Bell La Plata, ciudad (Buenos Aires), alcorque de acera. 
asociación de Valencia (España), la comunidad hallada suele ser muy pobre en especies, dominada por un estrato bajo, y de fenología invierno-primaveral. En la mayor parte de las formaciones del área estudiada predominan, en los ecosistemas naturales, las especies perennes estivales, no nitrófilas y que responden a una estacionalidad hídrica con lluvias principales durante el estío. Sólo en el oeste (Mendoza) los terófitos dominan sobre las perennes en la vegetación natural, pero no hay efímeras de invierno (Morello, 1958).

La posibilidad de que comunidades anuales tempranas y nitrófilas puedan desarrollarse en el área, depende casi exclusivamente del suministro de agua y de la nitrificación que pueden encontrar en el ámbito urbano. En consecuencia, la comunidad puede considerarse en cierto modo como hidrófila (azonal) dentro de un contexto zonal semiárido.

Comparte con la asociación descrita para Valencia una combinación estadística similar entre Poa annua, Coronopus didymus y Sisymbrium irio. Pero, a diferencia de ésta, el terófito Bromus catharticus se presentó en al menos un inventario en todas las ciudades que comprendió el trabajo y Cynodon dactylon también fue una compañera de importancia. Dos de las acompañantes presentaron un grado de presencia similar al de la asociación descrita por Carretero y Aguilella: Conyza bonariensis y Amaranthus muricatus. El resto de las especies presentó patrones diferentes.

De las 13 especies que figuran en los inventarios originales del PooCoronopodetum didymi, doce se encuentran registradas en al menos una de las provincias que abarcó el estudio. La única que no cuenta con citas para la región es Diplotaxis erucoides (L.) DC. (Zuloaga \& Morrone, 1999). Además, dos de las acompañantes del inventario tipo:'Amaranthus muricatus y
Aster squamatus son nativas y de amplia distribución en Argentina (Zuloaga \& Morrone, op. cit.).

Las especies características para los sintáxones de la clase presentes al menos en una de las provincias fueron los siguientes:

Características de la Clase PolygonoPoetea annuae y orden Polygono arenastriPoetalia annuae: Coronopus didymus*, Cotula australis, Sagina apetala, Poa annua, y Polygonum aviculare.

Alianza Polycarpion tetraphylli RivasMartínez 1975: Polycarpon tetraphylllum, y Soliva stolonifera*.

(Los táxones marcados con un asterisco son considerados nativos en Argentina).

II. Sucesionales o dinámicos. Carretero y Aguilella (1995) señalan para la asociación relacionada, que en las zonas rurales está en estrecho contacto con las comunidades arvenses de cultivos de regadío (DiplotaxioUrticion), marcando un tránsito hacia las mismas. Debido a que los inventarios del presente trabajo sólo incluyeron áreas urbanas, no se pueden realizar inferencias acerca del comportamiento sucesional en zonas agrícolas. No obstante, las dos especies características de la comunidad se citan como malezas de diversos cultivos (Marzocca, 1979) donde son frecuentes las especies características de varios sintáxones del Orden Solano nigri-Polygonetalia convolvulii: Cyperus rotundus, Amaranthus albus, Polygonum persicaria, Fumaria spp, Bidens subalternans, etc.

Una periodicidad estacional, consistente en un reemplazo de la comunidad durante el estío por otra dominada por Alternanthera pungens, no fue señalada para la asociación de Valencia.

III. Históricos y antropogénicos y del hábitat. Estos caracteres pueden considerarse similares, ya que los hábitats 
en los que se desarrolla coinciden con los del Poo annuae-Coronopodetum didymi y son principalmente los alcorques de aceras y bordes de caminos y aceras. Además en ambos casos la comunidad se instala y se mantiene por efecto de una intensa acción antropozoogénica.

IV. Corológicos. Estos caracteres son los que presentaron la mayor diferencia, puesto que si bien Carretero y Aguilella (1995) sugieren una distribución más amplia para la comunidad de Valencia, la alianza que la incluye: el Polycarpion tetraphylli, se encuentra ligado al areal mediterráneo (Rivas-Martínez et al., 2002). Sin embargo, Polycarpon tetraphyllum, la especie que da nombre a la alianza, es frecuente como adventicia en comunidades ruderales de Argentina, desde el nivel del mar a los 1000 $\mathrm{m}$, como también en países limítrofes: Brasil
Chile y Uruguay (Zuloaga y Morrone, 1999) y es considerada en la mayor parte de las floras como cosmopolita o subcosmopolita. Comunidades fragmentarias del Polycarpion, dominadas por Policarpon tetraphyllum, en ocasiones asociada a Sagina apetala, fueron observadas en varias ocasiones en intersticios de aceras del área estudiada.

\section{Análisis Twinspan}

Los agrupamientos principales de inventarios obtenidos con Twinspan muestran una estrecha relación entre la comunidad hallada (BCD) y el PCD (fig. 2). La similitud es mayor entre estos grupos de inventarios que entre los de PCD y PCS, este último característico de suelos con texturas más finas, menos permeables. También resultó mayor que la que existió entre las dos comunidades de Argentina BCD

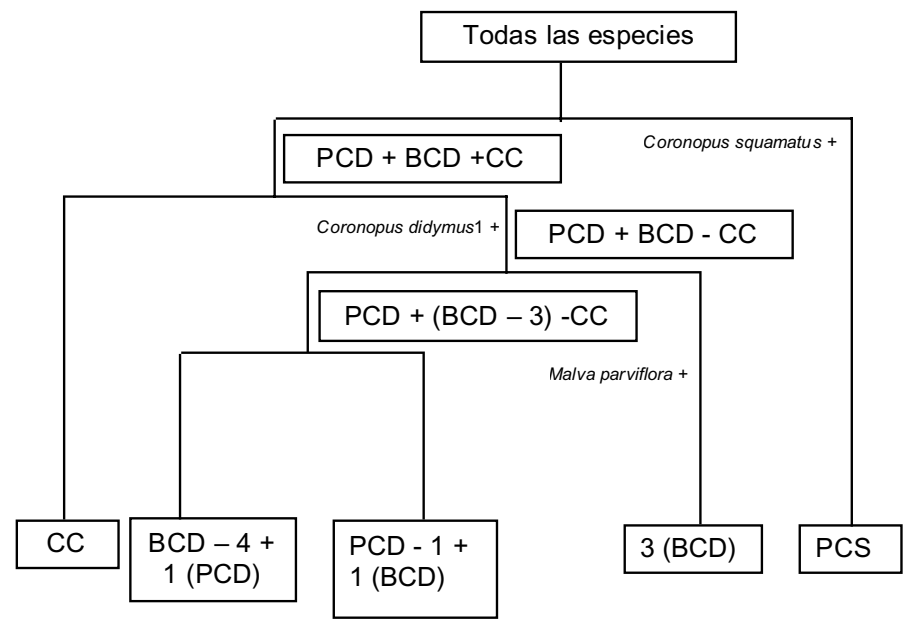

Figura 2. Resultados de Twinspan con las especies indicadoras de grupo. PCD: Poo annuaeCoronopodetum didymi; BCD: Bromo cathartici-Coronopodetum didymi; PCS: Poo annuaeCoronopodetum squamati; $\mathrm{CC}$ : Comunidad de Cotula australis; $\mathrm{BCD}-4=\mathrm{BCD}$ menos 4 inventarios; $+1(\mathrm{PCD})=$ más 1 inventario de PCD; PCD- $1=$ PCD menos un inventario $+1(\mathrm{BCD})=$ más 1 inventario de $\mathrm{BCD} ; 3$ (BCD): tres inventarios de $\mathrm{BCD}$. Results of Twinspan with the indicative species of group. PCD: Poo annuae-Coronopodetum didymi; BCD: Bromo cathartici-Coronopodetum didymi; PCS: Poo annuae- Coronopodetum squamati; $C C$ : Cotula australis community; $B C D-4=B C D-4$ relevés; $+1(P C D)=$ with 1 relevé of $P C D ; P C D-1=P C D-1$ relevé; $+1(B C D)=$ with 1 relevé of $B C D ; 3$ $(B C D)$ : three relevés of $B C D$. 
y CC.

Los inventarios de BCD y PCD aparecen como un bloque conjunto $\mathrm{y}$ diferenciado en primer término de los del PCS y de los de CC en segundo término. Las divisiones posteriores separan finalmente a siete inventarios del BCD más uno del PCD en un mismo bloque y a todos menos uno de los del PCD más uno del BCD en otro bloque, indicando la gran similitud ya señalada en el párrafo anterior.

\section{CONCLUSIONES}

Sobre la base del análisis y teniendo en cuenta las restricciones corológicas y las diferencias sucesionales, se consideró que la comunidad hallada constituye una nueva asociación: el Bromo catharticiCoronopodetum didymi Moglia ass. nova, (typus ass. inv. 2, tab. 2), vicariante neotropical, chaqueña del Poo annuaeCoronopetum didymi, con la cual se encuentra estrechamente relacionada. Por lo tanto, correspondería situarla dentro de la alianza Policarpyon tetraphylli, que de esta manera ampliaría su areal hasta América del Sur (Argentina). La descripción de esta nueva asociación se corresponde con la realizada para la comunidad.

La destrucción acelerada de la vegetación natural por parte del hombre, y la nitrificación asociada a la acción antropozoógena, conducen a un aumento constante del territorio ocupado por la vegetación nitrófila en todo el mundo. Y si bien, la completa homogeneización de las biotas parece ser improbable (Collins et al. 2000), la flora de las ciudades parece seguir esta tendencia.

La presencia de comunidades con dominancia de terófitas nitrófilas, de ciclo inverno-primaveral, en las zonas del área estudiada donde la vegetación nativa es predominantemente perenne y de ciclo estival, apoya la hipótesis de que la vegetación urbana sigue un patrón común en las ciudades. El mismo estaría determinado por los condicionantes antropozoogénicos, similares en diferentes ciudades del mundo, que resultan en una flora y vegetación, relativamente independientes de los factores ecológicos naturales propios de cada región. Así, las diferencias en las condiciones de fotoperíodo, estacionalidad hídrica y temperatura, que afectan al ciclo estacional de las comunidades, se disipan en el ambiente urbano. En consecuencia, el conjunto de especies urbanícolas, como también las asociaciones que forman, llegan a ser muy similares en regiones distantes del mundo.

Debido al carácter ubicuo y cosmopolita propio de los sintáxones nitrófilos, las «globalizaciones sintaxonómicas» podrían resultar más efectivas que las fragmentaciones, en lo que a Ecología urbana se refiere. En consecuencia, se debería evitar el uso de especies de amplia distribución como características de sintáxones de areal restringido, que constituye un impedimento para el desarrollo y crecimiento de la Fitosociología a lo largo y ancho del mundo.

El reconocimiento de conjuntos de especies formando parte de comunidades con las mismas características en sitios tan distantes del mundo es una prueba que confirma la validez y actualidad de la, en ocasiones discutida, Fitosociología como ciencia.

\section{BIBLIOGRAFÍA}

CABRERA, A. L. -1976- Regiones Fitogeográficas Argentinas. In: Kugler, W. F. (ed.). Encicl. Argent. Agric. y Jard. 2(1): 1-85

CARRETERO, J. L. y A. AGUILELLA -1995Flora y vegetación nitrófilas del término municipal de la ciudad de Valencia. Adjuntament de València. 233 pp.

COQUILLAT, M. -1951- Sur les plantes les plus 
comunes à la surface du globe. Bull. Soc. Mens. Linnéene Lyon 20: 165-170.

COLlins, M. D., D. P. VÁZQUEZ y N. J. SANDERS -2000- Species-area curves, homogenization and the loss global diversity. Evol. Ecol. Res. 4: 457-464.

FLORGÅD, C. -2004- Preservation of indigenous vegetation in urban areas -an introduction. Editorial. Landscape Urban Plann. 68(4): 343-345.

GÉHU, J. M. y S. RIVAS-MARTÍNEZ -1981Notions fondamentales de Phytosociologie. In: H. Diershke (ed.): Syntaxonomie, Ber. Intern. Symposien IVV., pp 5-33. J. Cramer, Vaduz.

HILL, M. O. -1994- Decorana and Twinspan, for ordination and classification of multivariate species data: a new edition together with supporting programs, in Fortran 77. Institute of terrestrial Ecology, Huntingdon.

KENT, M., R. A. STEVENS \& L. ZHANG -1999Urban plant ecology patterns and processes: a case study of the flora of the City of Plymouth, Devon, U. K. J. Biogeogr. 26: 1281-1289.

MARTÍNEZ CARRETERO, E. -1993Sintaxones arvenses, ruderales, adventicios, presentes en la vegetación argentina. Multequina 2: 195-200.

MARZOCCA, A. -1979- Manual de malezas. Hemisferio Sur, Buenos Aires. 566 pp.

MUELLER-DOMBOIS, D. \& H. ELLEMBERG -1974-. Aims and methods of vegetation ecology. John Willey \& Sons, New York, 547 pp.

MORELLO, J. -1958- La provincia fitogeográfica del monte. Opera Lilloana 2: 1-155.

PYSEK, P. -1995-Approaches to studying spontaneous settlements flora and vegetation in central Europe: a review. In: H. Sukkop, M. Numata \& H. Huber (eds.): Urban Ecology as the basis of urban planning, pp. 23-39. SPB Publishing, The Hague.

RIVAS-MARTÍNEZ, S. -1977- Datos sobre la vegetación nitrófila española. Acta. Bot. Malacitana 3: 159-167.

RIVAS-MARTÍNEZ, S. -1996- Geobotánica y climatología. Discurso del acto de Investidura como Dr. H. C. Universidad de Granada, Granada. 12 pp.
RIVAS-MARTÍNEZ, S., T. E DÍAZ, F. FERNÁNDEZ-GONZÁLEZ, F. IZCO, J. LOIDI, M. LOUSÁ \& A. PENAS -2002Vascular Plant communities of Spain and Portugal. Addenda to the syntaxonomical checklist of 2001. Part II. Itinera Geobot. 15(2): 478-479.

RUIZ LEAL, A. -1972- Flora popular mendocina. Deserta 3: 1-299.

TREPL, L. -1995- Towards a theory of urban biocoenoses, some hypotheses and research questions. In: H. Sukkop, M. Numata \& H. Huber (eds.): Urban Ecology as the basis of urban planning, pp. 3-21. SPB Publishing, The Hague.

WITTING, R. -1989- Metodische Probleme der Bestandsaufnahme der spontanen Flora und Vegetation von Städten. Braun-Blanquetia 3: 21-28.

ZULOAGA, F. O. y O. MORRONE (eds) -1996Catálogo de las plantas vasculares de la República Argentina I. Monogr. Syst. Bot. Missouri Bot. Gard. 60: 1-323.

ZULOAGA, F. O., E. G. NICORA, Z. E. RÚGOLO DE AGRASAR, O. MORRONE, J. PENSIERO y A. M. CIALDELLA -1994Catálogo de la Familia Poaceae en la República Argentina. Monogr. Syst. Bot. Missouri Bot. Gard. 47: 1-178.

ZULOAGA, F. O. y O. MORRONE -1999Catálogo de las plantas vasculares de la República Argentina II. - Monogr. Syst. Bot. Missouri Bot. Gard. 74: 1-1269.

WEBER, H. E., J. MORAVEC y J. P. THREURILLAT -2000- International Code of Phytosociological Nomenclature. 3rd edition. J. Veg. Sci. 11: 739-768.

Aceptado para su publicación en septiembre de 2006

Dirección de la autora. Área Biología, Facultad de Química Bioquímica, Farmacia y Ciencias Biológicas, Universidad Nacional de San Luis, Chacabuco y Pedernera, CP: 5700, San Luis (capital), San Luis, Argentina. E-mail: mmmoglia@unsl.edu.ar 